\title{
INFORMATION RETRIEVAL FROM TEXT
}

\author{
Apeksha Bhargava ${ }^{1}$ and Prof Sri Khetwat Saritha ${ }^{2}$ \\ ${ }^{1}$ Department of CSE And IT, Maulana Azad National Institute of Technology Bhopal \\ Madhya Pradesh India \\ apekkshabhargava85@gmail.com \\ ${ }^{2}$ Department of CSE And IT, Maulana Azad National Institute of Technology Bhopal \\ Madhya Pradesh India \\ sk.manitegmail.com
}

\begin{abstract}
With the rapidly increasing growth in the field of internet and web usage, it has become essential to use a certain specific powerful tool, which should be capable to analyze and rank all these available reviews/opinion on the web/Internet. In this paper we have propose a new and effective approach which uses a powerful sentiment analysis procedure which will be based on an ontological adjustment and arrangements. This study also aims to understand pos tag order to get detailed observation for any review or opinion, it also helps in identifying all present positive /Negative sentiments and suggest a proper sentence inclination. For this we have used reviews available on internet regarding Nokia and Stanford parser for the purpose or pos tagging.
\end{abstract}

\section{KEYWORDS}

Morphology, Association, Target, Implicit/Explicit conversion

\section{INTRODUCTION}

Sentiment or emotion is a thought or view of viewer that he wants to express towards something. Sentiment analysis is the process of extracting these emotion or sentiments. Sentiment analysis is the process of natural language processing (NLP) to extract the emotion from the text. The main task of sentiment analysis is to identify implicit and explicit emotion from the given document. Information science follows two main aspects which are facts and opinion. Facts deals with the exact detailing and opinion can be understand as someone's' thought, review or reaction about any product which has been launched recently. Opinions compromises of positive or negative adjectives and which reflects whether the product is purchasable or not or what impact it has on masses. Sentiment Analysis can also merely be termed as Opinion mining, this study of sentiments (positive /Negative) helps to find opinion inclination and also help to form a final opinion about the product .opinion can be put under two main categories: Direct comment or Comparisons. Direct opinion is what user / review thinks about the product. E.g. "NOKIA is not an extra ordinary mobile but always comes in parallel with latest trends, where as Comparison is a form of opinion which tells us which one is better between two products etc.

In section 1 related work has been introduced. In section 2 proposed approach is discussed which include input, analyzing of reviews (POS Tagging), section 3 includes figure description, section 4 includes association/ relationship between nodes, section 5 includes conclusion. 


\section{RELATED WORK}

Sentiment analysis is the process to identify the emotion which an opinion contains. Too much work has been done in this field. Sentiment analysis, emotion detection feature extraction are the fields of text mining. Feature extraction method is applied on the reviews provided by consumer. These comment could be short (one line) or it could be multiline comments. The new user could not make decision about a product or services of an organization by reading all these comments available on sites. To make decision making easy lots of work has been done. These approaches are lexicon based. Some approaches are machine learning based.

\subsection{Lexicon}

Lexicon is the collection of words of a specific domain. It is a group of words which worked as dictionary in specific domain. Some other type of dictionaries is also used in sentiment analysis. Many lexicon based approaches are there to identify the sentiments. It is a group of words which worked as dictionary in specific domain. Some other type of dictionaries is also used in sentiment analysis. SO-calculator, Sentiwordnet, WordNet and SentiStrength used in the lexicon based sentiment analysis.

\subsection{Corpus}

This uses a large collection of text for analysis known as corpus. It may contain text in single or multiple languages. Its plural form is known as corpora. Corpus is of two type open corpus and closed corpus. Open corpus could be limited in specific domain. Closed corpus may claim of containing of all or near about all related text of a specific domain.

In [1] system for voice of customer analysis is proposed, which will produce strong rules to help organization to take business decisions. It has used association mining. Association rule shows relationship between two or more entities.

Support s of the rule is defined as (XUY)/ 1 Tl confidence of the rule is defined as (XUY)/ (X) For example consider the rule $\{1,2\}->\{3\}$ i.e. items 1,2 implies 3

The support of a rule is important, since it shows how many times the rule is in the transactions, means it shows the frequency of the rule in transaction. The rules which have small Support value are of less importance in the transaction, because they do not give any use full information. Therefore the algorithm eliminates the candidates of less support than the threshold value. Often the value of minimum support is defined by user.

In [2] for feature selection one of the intuitively simplest metrics is the calculated expected accuracy for a simple classier built to identify a single feature. It can be calculated by taking the difference between the true positives and the false positives to determine how many times the correct feature is selected. The balanced accuracy takes the difference between the true positive rate and the false positive rate rather than the numbers of true and false positives. The main advantage of this is the removal of a strong preference for a low false positive rate.

In [3] and [4] work on correlation based feature selection has been done. Correlation based calculation can be generally divided into two categories: one is linear correlation such as linear correlation coefficient, Pearson product moment correlation. The other one is based on information theory, such as entropy. Here, correlation coefficient $r$ as the measurement of correlation for features. 
Let's suppose that the feature set is $S[F 1, F 2, \ldots, F N], \mathrm{N}$ is the total number of features, $\mathrm{C}$ is category attribute. Here, two kinds of correlations for input features. The strength of correlation between a feature and the classification label determines the classification performance. The stronger the correlation is, the better the classification performance is. Therefore, firstly we should remove the features uncorrelated with class attribute in order to find an optimal feature subset.

The proposed method is also working to identify the features from the given reviews. Stanford parser has been used here for morphological analysis. WordNet has been applied to identify the synset used in these reviews. WordNet is a NLP (natural language processing) tool, which contain the lexicon.

\section{Proposed APPROACH}

It is difficult for a new customer to make decision about a product by reading views. Our method makes easy the decision making of the new customer by extracting the emotion from the reviews.

\subsection{Data Source}

We are working on the data set of NOKIA, Which contains both the positive as well as negative review about the nokia. The search function reads the string from both the ends, and generates the left and right ontological tree respectively. In the final step both ontological trees get merged and final result tree has been generated. The tree has root value which is the target of the given opinion.

\subsection{Morphological Analysis}

Morphological analysis is the main part of Natural Language Processing. Any comment / Review or opinion available on the internet may have a complex structure and require high efforts to understand first, in terms of sentence analyzing here we are focusing on understanding about grammar part. This analysis proceeds through POS tagging and sentence splitting which is also known as tokenization.

\subsubsection{Pos Tagging}

A process of ranking all possible parts of speech like Noun, Pronoun, Verb, Adverb, Adjective etc is called Pos tagging. To analyze any sentence completely in its grammatical context STANFORD PARSE has been used.

Example

\begin{tabular}{|c|l|}
\hline Input & I LIKE MY NOKIA \\
\hline POS & $\begin{array}{l}\text { (I,PRP),(LIKE,VBZ),(MY,PRPS), } \\
\text { (NOKIA,NNP) }\end{array}$ \\
\hline
\end{tabular}


The parser parses all the strings. The database stores the Part of Speeches in the table form.

Here $\langle\mathrm{PRP}\rangle$ shows personal pronoun, $\langle\mathrm{VBZ}\rangle$ shows verb third person singular present, $\langle$ PRPS $>$ shows possessive pronoun, $\langle\mathrm{NNP}\rangle$ shows proper noun singular. For morphological analysis we are applying Stanford parser which parser the whole dataset. The POS tagging labels used include JJ for adjective, DT for determiner, VB for verb, $\mathrm{RB}$ for adverb, $\mathrm{NN}$ for noun, $\mathrm{CC}$ for coordinating conjunction, and $\mathrm{CD}$ for cardinal number.

The target value is based on the frequency count of the particular noun in the NN table.

\subsection{Sentence Splitting (Tokenization)}

It is the process of splitting given data into small units known as token, which could be Character, any number or any special character. It is also known as word segmentation.

\subsection{Implicit and explicit conversion}

In the public review the consumers talk about many feature of a particular product. They writes about the feature without specifying the product name again and again, whether they used some words like this, that, those, which, it etc .These all are indirectly indicating towards the target.

For example in the string

"I love my nokia phone; its touch is so nice."

In the above string the noun is NOKIA, which has frequency count 1 , but in the second part of the sentence pronoun its increases the frequency count by +1 of the NN nokia implicitly. The final ontological tree is as follows "IT' $\mathrm{S}$ " directly implies to the noun.

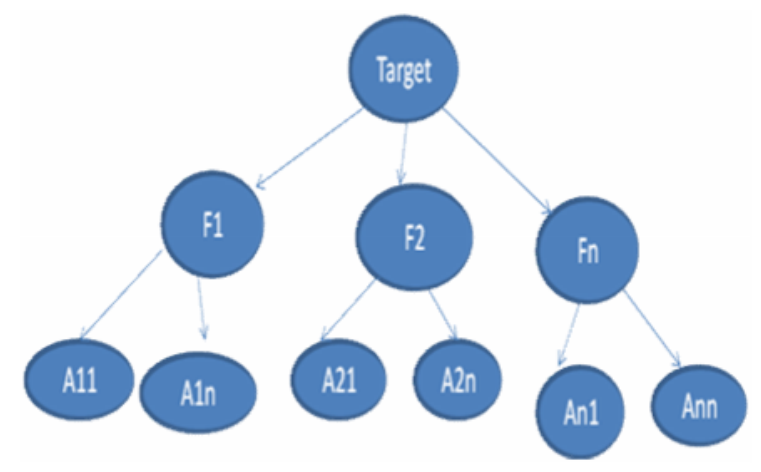

Figure: Final ontological tree

The above figure is the final ontological tree. In the figure Root node is showing the target.

$$
\text { E.g. Battery life of nokia is long. }
$$

In the figure F1, F2 and FN are the different features of the target. These features show the property of the target value. The features are extracted from the dataset. The last level containing values from A11 - An2.A11 and A12 are the adjectives Associated with the feature F1.A21 and 
A22 are the adjectives Associated with the feature F2. An1 and An2 are the adjectives Associated with the feature Fn. These all adjectives give polarity to the features at the second level of the tree. This polarity decides whether the reviews are positive or negative about the target. This result is based on the association between the target - feature value and between targets characteristics of that feature. An association shows the relationship/dependency between two levels of the tree. In the above tree the first node is showing the target value about which we are extracting the emotions/sentiments or information for the public reviews. The second level is keeping the record of the features of the target .These all features decides the market value of the target. The third and the last level keep the records of the all emotion about a target. These all emotion gives positive or negative polarity to the features, which decides the final market value of the target. The resulting ontological tree is based on the association between the two levels of the tree. Association shows the relationship between two entities. In the above figure targetfeature association is showing about how many features the reviewer giving their opinion. The number of features is extracted from the public reviews. The second level is showing the feature characteristics association of the target.

Steps of Tree Formation

1. Reviews are taken from sites.

2. POS tagging is applied on input strings.

Stanford parser is used for parsing the input string.

3. All features which are noun come at the second level.

4. All features that have some characteristics associated in the input string which comes at the third level.

5. These all features give polarity to the associated feature.

Final opinion for market hypothesis is calculated based on this polarity of features.

\section{CONCLUSIONS}

Information retrieval in text mining is very useful to summarize all the reviews or text available on the web sites. It is making decision easy for a new user or consumer about a product or services of any organization. To make decision making easy Morphological analysis is used. This approach helps to identify products features polarity.

\section{REFERENCES}

[1] Shubha S. Jain ,B.B. Meshram ,Munendra Singh "Voice of Customer Analysis using Parallel Association Rule Mining" Students' Conference on Electrical, Electronics and Computer Science, 978-1-4673-1515-9 ,2012 IEEE

[2] Nicolette Nicolosi Feature Selection Methods for Text Classification, November 7, 2008.

[3] Franc, ois Mairesse, Joseph Polifroni, Giuseppe Di Fabbrizio," can prosody inform sentiment analysis? experiments on short spoken reviews",ICASSP 2012 978-1-4673-0046-9, 2012 IEEE.

[4] Jinjie Huang, Ningning Huang, Luo Zhang, Hongmei Xu, "A method for feature selection based on thecorrelation analysis", 2012 International Conference on Measurement, Information and Control (MIC), 978-1-4577-1604-1, 2012 IEEE.

[5] G.Vinodhini, RM.Chandrasekaran "Sentiment Analysis and Opinion Mining: A Survey", Volume 2, Issue 6, June 2012, ISSN: 2277 128X, IJARCSSE.

[6] Huosong Xia, Min Tao,Yi Wang "Sentiment Text Classification of Customers Reviews on the Web Based on SVM, 2010 Sixth International Conference on Natural Computation (ICNC 2010), 2010 IEEE 


\section{Authors Profile}

Saritha Khethawat received the B.E.degree in Osmania University, Hyderabad,India. M.Tech. in Computer Science and Engineering from Maulana Azad National Institute of Technology, Bhopal, India.

Apeksha bhargava received the B.E. degree in Information Technology from RGPV University,Bhopal,India, in 2009.and M.Tech. in Computer Science and Engineering from Maulana Azad National Institute of Technology, Bhopal, India.
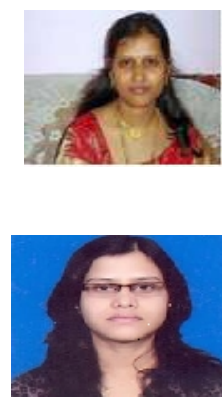